Test Setup for Characterisation of Biased Magnetic Hysteresis Loops in Power Electronic Applications

\author{
M. Luo, D. Dujic, and J. Allmeling
}

This material is posted here with permission of the IEEE. Such permission of the IEEE does not in any way imply IEEE endorsement of any of EPFL's products or services. Internal or personal use of this material is permitted. However, permission to reprint / republish this material for advertising or promotional purposes or for creating new collective works for resale or redistribution must be obtained from the IEEE by writing to pubs-permissions@ieee. org. By choosing to view this document, you agree to all provisions of the copyright laws protecting it. 


\section{Test Setup for Characterisation of Biased Magnetic Hysteresis Loops in Power Electronic Applications}

\author{
Min Luo and Drazen Dujic \\ Power Electronics Laboratory \\ École Polytechnique Fédérale de Lausanne (EPFL) \\ Lausanne CH-1015, Switzerland \\ min.luo@epfl.ch,drazen.dujic@epfl.ch
}

\author{
Jost Allmeling \\ Plexim GmbH \\ Zürich CH-8005, Switzerland \\ allmeling@plexim.com
}

\begin{abstract}
Hysteresis effect of core materials contributes significantly to the power loss and nonlinearity of the transformers and filter inductors in power electronic applications. For design or modeling of the magnetic components, information of the magnetic material's characteristic under the desired operation condition is usually required. In many common types of power converters the magnetic components undertake biased excitation, which leads to hysteresis loop with DC-offset on both magnetic field strength and flux density. This work proposes a test setup combining both linear amplifier and switching cells, which is able to conveniently generate magnetic hysteresis loops at arbitrary biased levels.
\end{abstract}

\section{INTRODUCTION}

The core material of isolation transformers or filter inductors used in power electronic converters exhibits hysteresis effect, which significantly contributes to the power loss and nonlinear inductivity of the magnetic components. In order to properly design the magnetic component in terms of core dimension, winding configuration or thermal condition, the core material characteristic on the B-H plane, or in other words, the hysteresis loops are usually required. For the measurement of hysteresis loops, the V-I approach introduced in the work of [1] is commonly adopted nowadays. This approach is improved in terms of measurement accuracy by several publications like [2] and [3] for power loss of symmetrical hysteresis loops.

In many types of power converters, unsymmetrical hysteresis loops with bias are also present, as in the case of a flyback DC-DC converter shown in Fig. 1(a) or a voltage source DC-AC inverter shown in Fig. 1(b). In order to characterise the biased hysteresis loops of the core material using a core sample, one option is to introduce an extra DC excitation. Authors of [4] installed a third winding supplied by DC voltage source and authors of [5] made use of two identical core samples. Aiming to imitate the operation conditions in power electronic converters under PWM excitation, [6] used a $\mathrm{H}$ bridge converter and the DC-biased excitation was generated via applying different duty cycles to the gate signals. In these publications, only the DC-offset on the field strength $H$ direction was considered and explicitly controlled during the measurement. However the bias of the hysteresis loops is also present on the flux density direction (vertical direction of the B-H plane in Fig. 1(a) and Fig. 1(b)). Authors of [7]
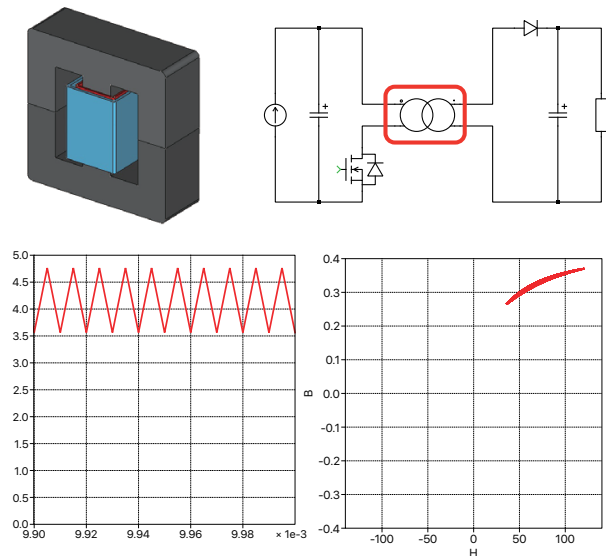

(a)
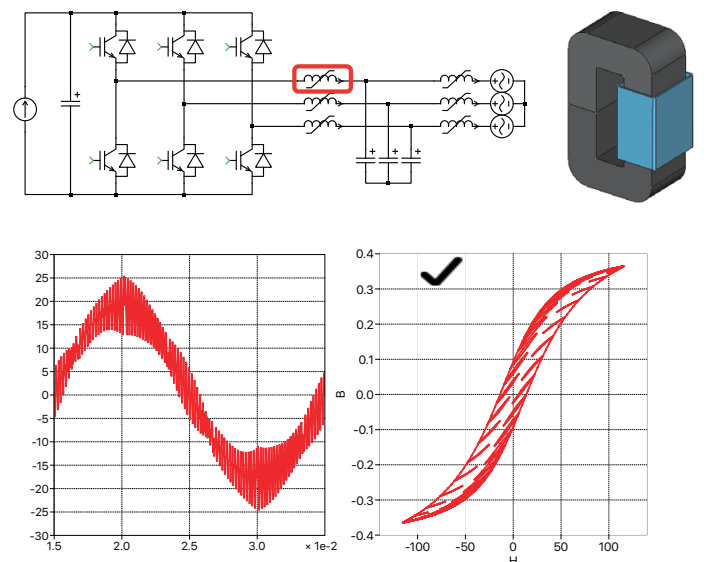

(b)

Fig. 1: Biased magnetic hysteresis loop as well as the corresponded magnetisation current waveform in power electronic applications (a) Simplified flyback converter; (b) Voltage source inverter.

and [8] have observed the "noncongruency" effect present in the real magnetic materials, that is: Even the minor hysteresis loops having the same range of field strength $H$ exhibit different shapes if they are located at different flux density 


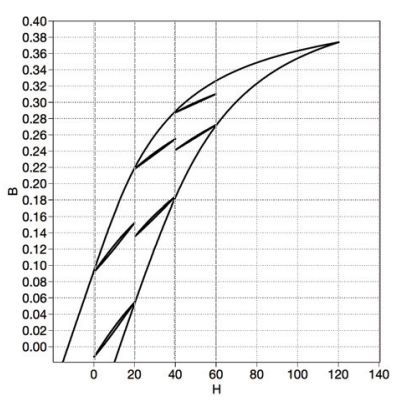

(a)

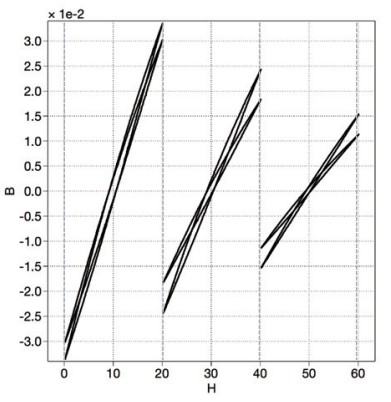

(b)
Fig. 2: Demonstration of noncongruency in reality of hysteresis loops in the same $H$ range and different $B$ positions (a) Biased hysteresis loop on their original $B$ positions; (b) Shape comparison after the bias of $B$ is artificially removed.

position on the B-H plane, depending on the magnetisation history, as has been demonstrated in Fig. 2(a) of the ferrite material N87. The difference is not only related to the enclosed loop area (determines the power loss) but also the equivalent permeability, which directly affect the dynamic behaviour of the magnetic component and need to be taken into account during design phase.

In order to characterise the hysteresis loops at different $B$ positions, authors [9] and [10] proposed to overlap a fundamental frequency- with a harmonic frequency sinusoidal voltage excitation. The fundamental frequency excitation accounts for generating a symmetrical major hysteresis loop and the harmonic frequency one results in biased minor loops attached on the major loop, so that the $B$ position of each biased loops can be identified. However using sinusoidal harmonic excitation it is not easy to quantitatively control the $B$ position of the biased loops, also in power electronic applications the biased loops are initiated by PWM- instead of sinusoidal excitation. This work aims to facilitate the characterisation of biased hysteresis loops by means of accurately controlling the $B$ position as well as equivalent switching frequency for power electronic applications. For that purpose, a highly flexible low power characterisation setup is developed, as described next, allowing for great degree of freedom in driving a magnetic core sample into a desired operation point.

\section{PROPOSED APPROACH}

The basic circuit configuration of the proposed test setup is depicted in Fig. 3(a), which is constructed based on the V-I method introduced by [1]. The core sample is equipped with two windings: The primary winding is connected to the excitation and the current $I_{p}$ is measured, which is converted into the field strength $H$. The secondary winding is left open and the voltage $V_{s}$ is obtained, which is integrated in time domain and converted into the flux density $B$.

Different from the existing publications, the primary winding in the proposed test setup is supplied by a sinusoidal-

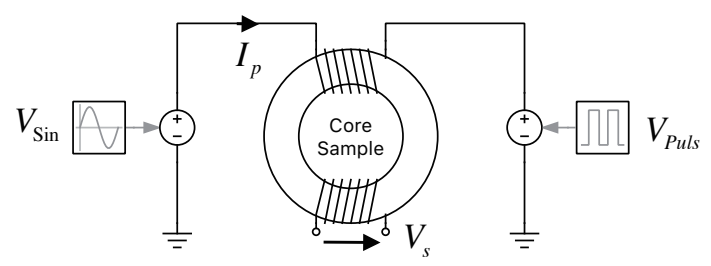

(a)

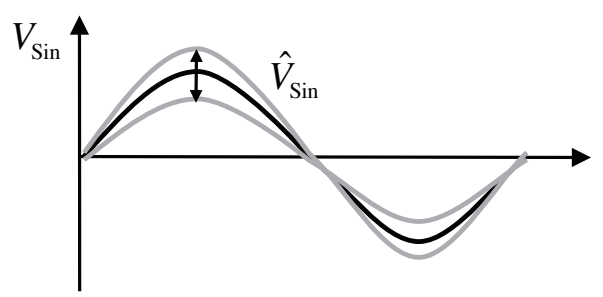

(b)

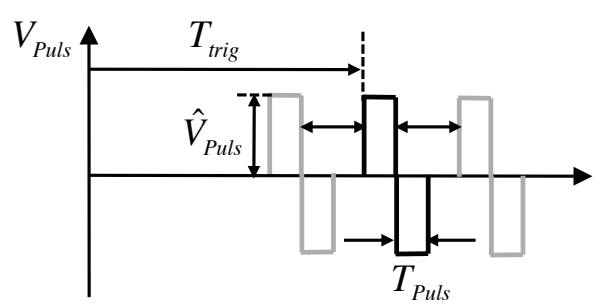

(c)

Fig. 3: Basic configuration of the proposed test setup (a) Schematic; (b) Voltage waveform of the sinusoidal excitation; (c) Voltage waveform of the pulsed excitation.

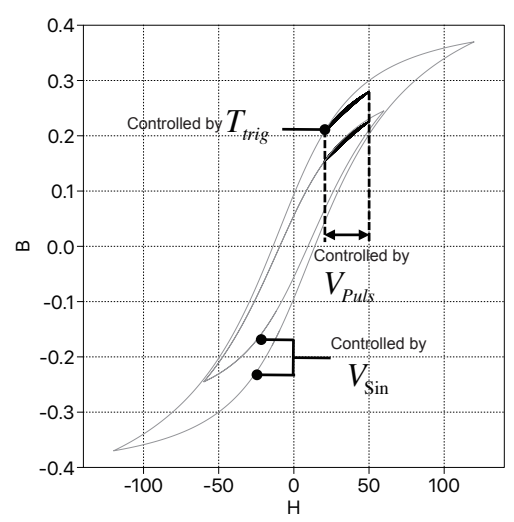

Fig. 4: Biased hysteresis loop located at desired field strength range $H$ and flux density $B$ position via adjusting the amplitude of the sinusoidal- and pulsed excitation, as well as the phase delay of the pulsed excitation.

and a square-wave pulsed voltage source, which allows to setup the biased hysteresis loop at arbitrary operation point: When the primary winding is supplied solely by the sinusoidal 
voltage source (Fig. 3(b)), a symmetrical hysteresis loop with large amplitude is generated. If the pulsed voltage source is applied at the same time (Fig. 3(c)), a biased hysteresis loop is present attaching on the symmetrical loop. As demonstrated in Fig. 4, on a symmetrical loop generated by the sinusoidal excitation, the $H$ position where the biased loop is initiated can be explicitly controlled by the phase delay $T_{\text {trig }}$ (Fig. 3(c)) of the pulsed excitation, with respect to the sinusoidal excitation. The width of the pulsed voltage $T_{\text {puls }}$ can be configured to setup the desired equivalent switching frequency for the biased loop. With given $T_{P u l s}$, the field strength $H$ range of the biased loop can be adjusted by tunning the amplitude $T_{P u l s}$ of the pulsed voltage. If another amplitude $\hat{V}_{\text {Sin }}$ of the sinusoidal excitation is applied which changes the symmetrical hysteresis loop's size, the biased loop in the same $H$ range can be located at any desired $B$ positions. In this way, the proposed test setup allows to characterise the materials's B-H property at arbitrary operating conditions.

Please note that it is important to setup the period of the pulsed voltage excitation to be equal to that of the sinusoidal one and keep both excitations always synchronised, so that the biased loop stay at the same location on the B-H plane.

\section{HARDWARE REALISATION}

The hardware realisation of the proposed test setup is illustrated in Fig. 5(a) and Fig. 5(b), which is composed of the following parts:

- A power amplifier of type LM3886 to generate the sinusoidal excitation.

- A single phase T-shape MOSFET bridge to provide three-level square-wave pulsed excitation, the DC side of which is supplied by another two power amplifiers.

- The primary current is measured by a shunt resistor in combination with fully differential instrumentation amplifier.

- The secondary voltage is measured using resistive voltage divider.

- A control unit (PLECS RT-Box) is equipped to provide reference signal for the power amplifiers, generate gate signal for the MOSFET bridge as well as process the measurements.

Since the test setup is supposed to characterise the material characteristic using small core samples, only low voltage output in the range $\pm 24 \mathrm{~V}$ and low current up to $6 \mathrm{~A}$ are required. The MOSFETs of the T-shape bridge are driven by carrier based PWM, in order to generate the pulsed voltage waveform shown in Fig. 3(c), the carrier as well as modulation index are configured as demonstrated in Fig. 6. The four switches are divided into two groups - $Q 1 \& Q 3$ and $Q 2 \& Q 4$, both of which have the same saw-tooth carrier. The two switches inside each group have the same modulation index while opposite polarity. The modulation index $m_{1}$ of the group $Q 1 \& Q 3$ and $m_{2}$ of Q2\&Q4 fullfills $m_{1}=1-m_{2}$.

\section{EXPERIMENTAL RESULTS}

For demonstration purpose, test result has been produced for ferrite material $\mathrm{N} 87$ from TDK, where the toroidal core of size code "R $41.8 \times 26.2 \times 12.5$ " is taken as sample. The turns number of the primary- and secondary windings are both eight. Two test schemes has been carried out, where the amplitude of the sinusoidal excitation has been configured to make the amplitude of the symmetrical hysteresis loop to be $\hat{H}=45 \mathrm{~A} / \mathrm{m}$ and $\hat{H}=60 \mathrm{~A} / \mathrm{m}$, respectively. The frequency of the sinusoidal excitation has been configured to be $200 \mathrm{~Hz}$. The phase delay $T_{\text {trig }}$ and amplitude of the pulse excitation are adjusted so that the biased minor loop in both test schemes are within the same field strength range $20 \sim 40 \mathrm{~A} / \mathrm{m}$. The pulse width $T_{\text {Puls }}$ is configured to be $0.25 \mathrm{~ms}$, yields $2 \mathrm{kHz}$ equivalent switching frequency. The measured hysteresis loop (including the symmetrical- and biased loops) as well as the time domain waveform of the primary winding current and

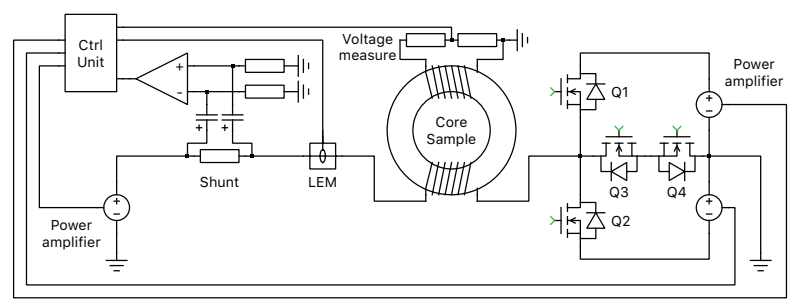

(a)

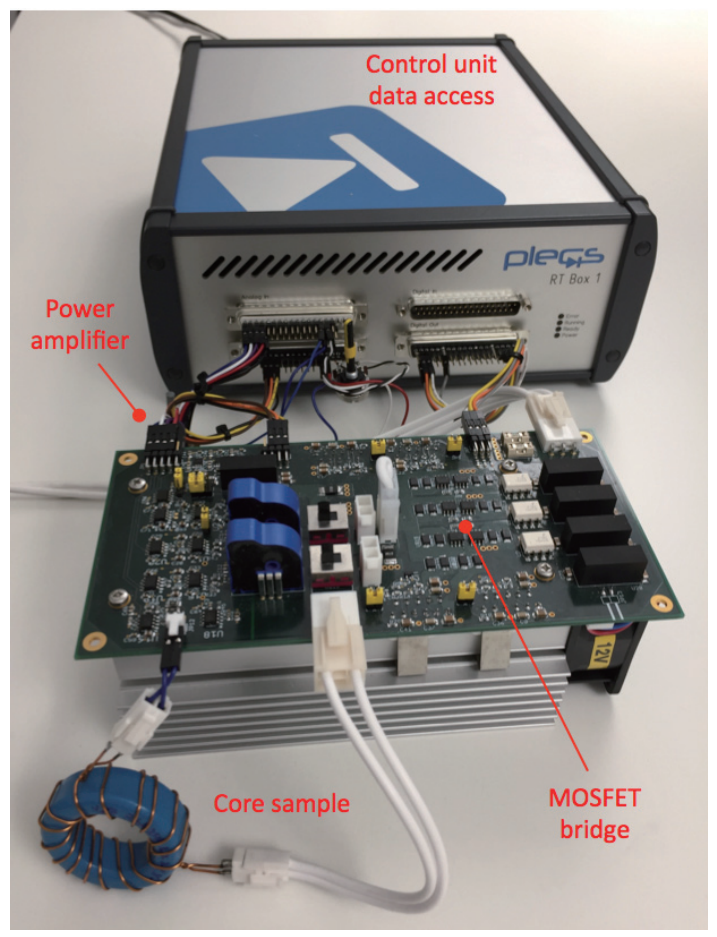

(b)

Fig. 5: Implementation of the proposed test setup (a) Hardware setup; (b) Circuit schematic. 


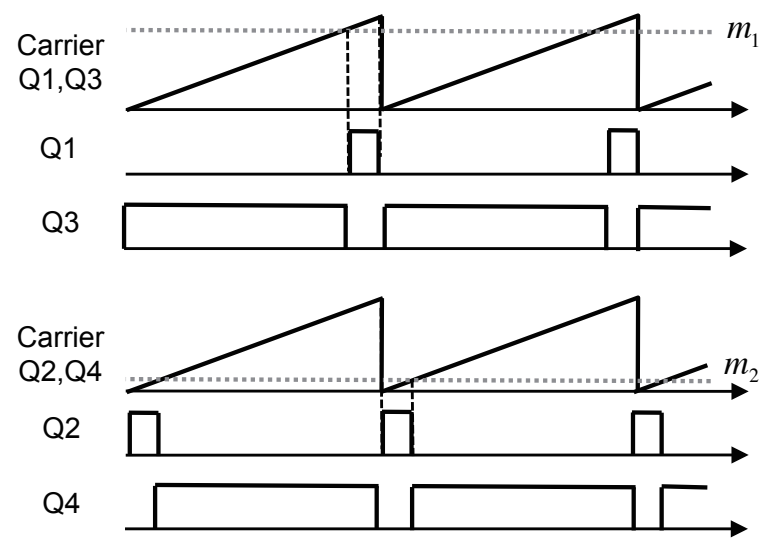

Fig. 6: Saw-tooth carrier based PWM generation of the T-shape MOSFET bridge.

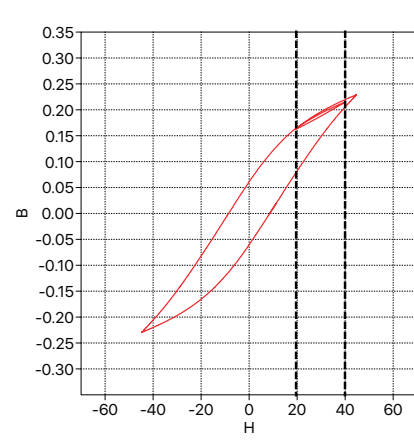

(a)

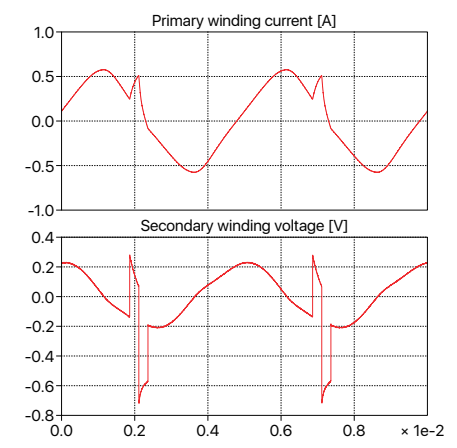

(b)
Fig. 7: Biased minor loop in the range $20 \sim 40 A / m$ with symmetrical loop's amplitude $45 \mathrm{~A} / \mathrm{m}$ and equivalent switching frequency $2 \mathrm{kHz}$ (a) B-H characteristic; (b) Time domain waveform of the primary current and secondary voltage.

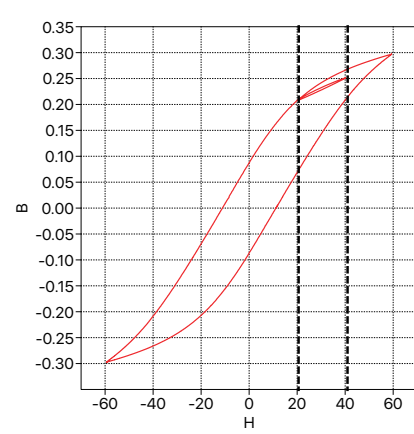

(a)

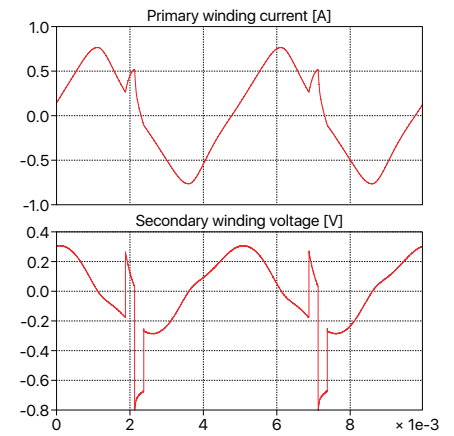

(b)
Fig. 8: Biased minor loop in the range $20 \sim 40 A / m$ with symmetrical loop's amplitude $60 \mathrm{~A} / \mathrm{m}$ and equivalent switching frequency $2 k H z$ (a) B-H characteristic; (b) Time domain waveform of the primary current and secondary voltage.

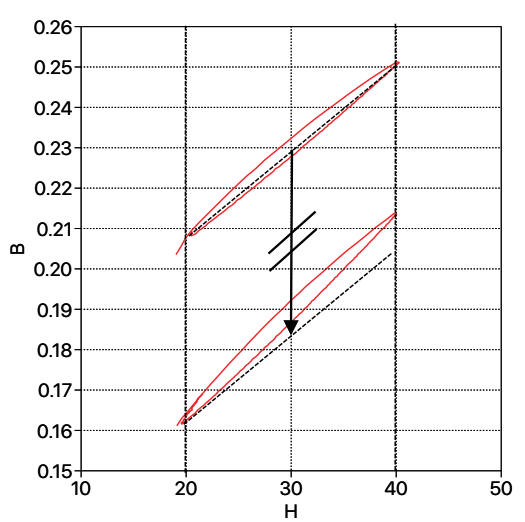

Fig. 9: Comparison of the two biased loop at different flux density $B$ positions, where obvious difference on equivalent permeability can be observed.

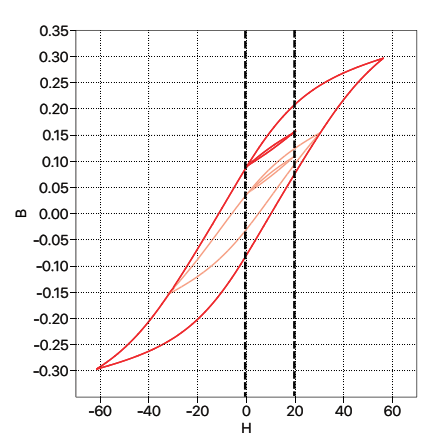

(a)

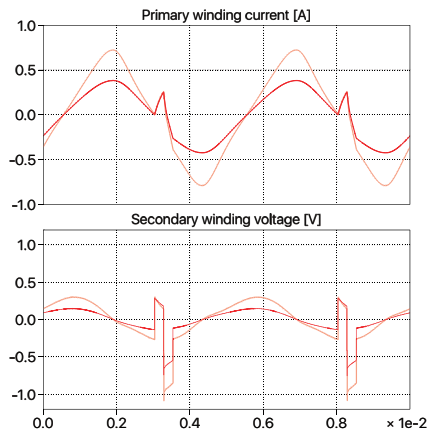

(b)
Fig. 10: Biased minor loops within $0 \sim 20 A / m$ with symmetrical loop's amplitude $60 \mathrm{~A} / \mathrm{m}$ and $30 \mathrm{~A} / \mathrm{m}$, equivalent switching frequency $2 k H z$ (a) B-H characteristic; (b) Time domain waveform of the primary current and secondary voltage.

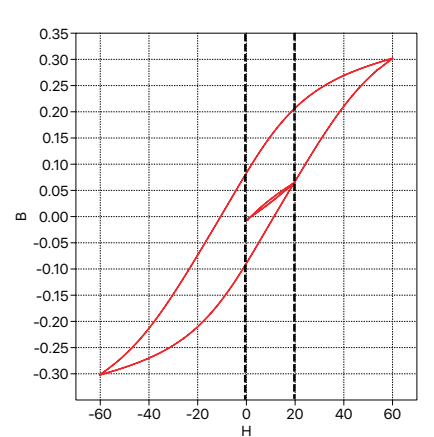

(a)
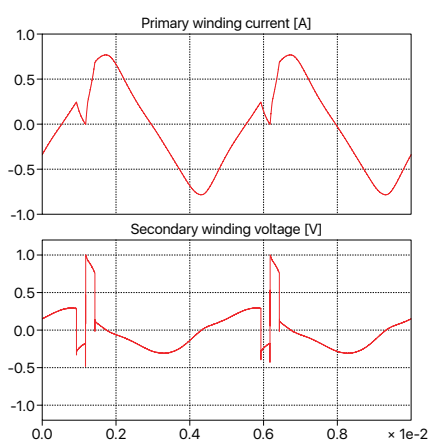

(b)
Fig. 11: Biased minor loop within $0 \sim 20 \mathrm{~A} / \mathrm{m}$ initiated on the ascending branch of a symmetrical loop with amplitude equal to $60 \mathrm{~A} / \mathrm{m}$ (a) $\mathrm{B}-\mathrm{H}$ characteristic; (b) Time domain waveform of the primary current and secondary voltage. 


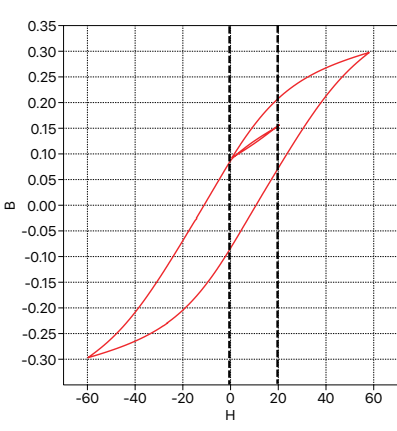

(a)

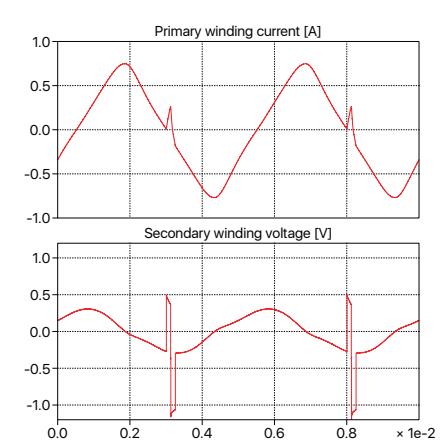

(b)

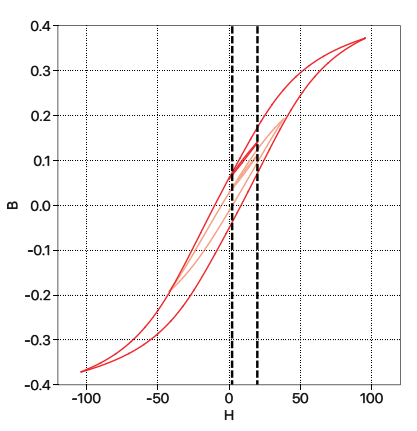

(a)

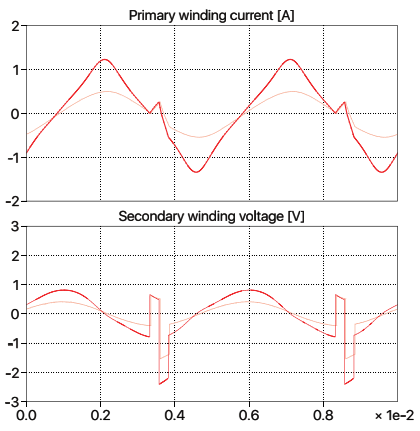

(b)
Fig. 12: Biased minor loop in the range $0 \sim 20 \mathrm{~A} / \mathrm{m}$ with equivalent switching frequency $4 k H z$ (a) B-H characteristic; (b) Time domain waveform of the primary current and secondary voltage.

secondary voltage in both test schemes are shown in Fig. 7(a) and Fig. 8(a), respectively. The minor loop on the B$\mathrm{H}$ plane corresponds to the triangular peak on the primary winding current waveform as an result of the pulsed-excitation provided by the T-bridge, which can be observed on the secondary winding voltage waveform. In Fig. 9, the two biased hysteresis loops obtained at different flux density $B$ positions are compared with each other, obvious difference can be observed on the equivalent permeability, or in other words, the slope of the virtual straight line connecting the two peaks of the loops.

By adjusting the phase delay of the gate signals $T_{\text {trig }}$ with respect to the sinusoidal excitation voltage, the $H$ range of the minor loops can be changed. In Fig. 10(a) and Fig. 10 (b), the measured minor loops within the field strength range $20 \sim 40 \mathrm{~A} / \mathrm{m}$ with different flux density bias are presented together with the corresponding time domain waveforms. Please note that the previous results are obtained when the minor loops are initiated on the descending branch of the symmetrical loop, if lower flux density bias is desired, the minor loop should be initiated on the ascending branch. For this purpose, the gate signals for the two switch groups $Q 1 \& Q 3$ and $Q 2 \& Q 4$ are exchanged. The test result of a minor loop initiated on the ascending branch of the symmetrical loop is presented in Fig. 11(a) and Fig. 11(b).

Higher equivalent switching frequency can be achieved via applying shorter pulse-width $T_{P u l s}$ and higher DC voltage $\hat{V}_{\text {Puls }}$. The measured minor loop within the field strength range $0 \sim 20 \mathrm{~A} / \mathrm{m}$ with a equivalent switching frequency of $4 \mathrm{kHz}$ as well as the corresponding primary winding current and secondary winding current are demonstrated in Fig. 12(a) and Fig. 12(b), respectively. In comparison to the secondary voltage waveform in Fig. 10(b), the duration of the pulse component is one half while the voltage is approximately doubled. If a duty cycle other than $50 \%$ is desired, the summation of the modulation indices $m_{1}$ and $m_{2}$ becomes higher than 1, while the DC supply voltages need to be trimmed as well so that the
Fig. 13: Biased minor loops in the range $0 \sim 20 \mathrm{~A} / \mathrm{m}$ of ferrite material $3 C 81$, equivalent switching frequency $2 k H z$ (a) B$\mathrm{H}$ characteristic; (b) Time domain waveform of the primary current and secondary voltage.

voltage-second products of the positive- and negative impulse remain the same.

At the end, another core sample $T X 51 / 32 / 19$ made of ferrite material 3C81 from Ferroxcube is characterised using the proposed approach. The minor loops in the field strength range $0 \sim 20 \mathrm{~A} / \mathrm{m}$ with different flux density bias are shown in Fig. 13(a). In comparison to the material N87, the large symmetrical hysteresis loop of 3C81 is significantly narrower. Considering the fact that the flux density range of the minor loops does not exceed the boundary of a large symmetrical major loop, the minor loops of $3 \mathrm{C} 81$ have limited range in the $B$ direction, so that the impact of the flux density bias on the shape of the minor loops is also lower.

\section{CONCLUSION}

This work proposes a new approach to characterise the biased hysteresis loop of core materials in power electronic applications. This approach combines continuous sinusoidaland switched PWM excitation together to imitate arbitrary operating conditions that take place in real power converter systems. The test can be easily carried out under low-voltage and low-current. Using this approach, the offset of the magnetic hysteresis loop on not only the field strength $H$ but also that on the flux density $B$ are accurately controlled, so that the influence of the historical magnetisation on the core loss and equivalent permeability can be clearly investigated. Also the equivalent switching frequency of the biased loop can be configured in a convenient way. Based on the measured material characteristic, the design and modelling of the magnetic component can be carried out in a more accurate way, in terms of meeting the THD requirement as well as optimising the system efficiency.

\section{ACKNOWLEDGMENT}

This project has been supported in the frame of the ECPE Joint Research Programme. 


\section{REFERENCES}

[1] D. Tan, J. L. Vollin, and S. M. Cuk, "A practical approach for magnetic core-loss characterization," in IEEE Transactions on Power Electronics, vol. 10 , no. 2,1995 , pp. 124-130.

[2] M. Mu, Q. Li, D. J. Gilham, F. C. Lee, and K. D. T. Ngo, "New core loss measurement method for high-frequency magnetic materials," in IEEE Transactions on Power Electronics, vol. 29, no. 8, 2014, pp. 4374-4381.

[3] D. Hou, M. Mu, F. C. Lee, and Q. Li, "New high-frequency core loss measurement method with practial cancellation concept," in IEEE Transactions on Power Electronics, vol. 32, no. 4, 2017, pp. 2987-2994.

[4] J. Reinert, A. Brockmeyer, and R. W. A. A. D. Doncker, "Calculation of losses in ferro- and ferritemagnetic materials based on the modified steinmetz equation," in IEEE Transactions on Industry Applications, vol. 37, no. 4, 2001, pp. 1055-1061.

[5] Y. Han and Y. Liu, "A practical transformer core loss measurement scheme for high-frequency power converter," in IEEE Transactions on Industrial Electronics, vol. 55, no. 2, 2008, pp. 941-948.

[6] J. Muehlethaler, J. Biela, J. W. Kolar, and A. Ecklebe, "Core losses under the dc bias condition based on steinmetz parameters," in IEEE Transactions on Power Electronics, vol. 27, no. 2, 2012, pp. 953-963.

[7] G. Kadar and E. D. Torre, "Hysteresis modeling: I. noncongruency," in IEEE Transactions on Magnetics, vol. 23, no. 5, 1987, pp. 2820-2822.

[8] M. Marracci and B. Tellini, "Hysteresis losses of minor loops versus temperature in mnzn ferrite," in IEEE Transactions on Magnetics, vol. 49, no. 6, 2013, pp. 2865-2869.

[9] M. Ibrahim, "M. ibrahim and p. pillay," in Core loss prediction in electrical machine laminations considering skin effect and minor hysteresis loop, vol. 49, no. 5, 2013, pp. 2061-2068.

[10] T. Taitoda, Y. Takahashi, and K. Fujiwara, "Iron loss estimation method for a general hysteresis loop with minor loops," in IEEE Transactions on Magnetics, vol. 51, no. 11, 2015. 